\title{
Through-Space Interaction: What, Where and How?
}

\author{
Junkai Liu, Haoke Zhang*, Lianrui Hu, Jun Wang, Jacky W. Y. Lam, Lluis Blancafort* and Ben Zhong \\ Tang*
}

\author{
Junkai Liu, Dr. Lianrui Hu, Dr. Jacky W. Y. Lam, Prof. Ben Zhong Tang \\ Department of Chemistry, Hong Kong Branch of Chinese National Engineering Research Center for Tissue Restoration and Reconstruction and Institute for \\ Advanced Study \\ The Hong Kong University of Science and Technology \\ Clear Water Bay, Kowloon, Hong Kong (China)
}

Prof. Ben Zhong Tang

Shenzhen Institute of Aggregate Science and Technology, School of Science and Engineering

The Chinese University of Hong Kong, Shenzhen, Guangdong 518172 (China)

Centre for Aggregation-induced emission, SCUT-HKUST Joint Research Laboratory, State Key Laboratory of Luminescent Materials and Device

South China University of Technology, Guangzhou 510640 (China)

AIE Institute, Guang Zhou Development District, Huangpu, Guangzhou 510530 (China)

E-mail: tangbenz@ust.hk

Prof. Haoke Zhang

MOE Key Laboratory of Macromolecular Synthesis of Functionalization, Department of Polymer Science and Engineering

Zhejiang University, Hangzhou 310027 (China)

E-mail: zhanghaoke@zju.edu.cn

Guangdong Provincial Key Laboratory of Luminescence from Molecular Aggregates

South China University of Technology, Guangzhou 510640 (China)

Dr. Jun Wang, Prof. Lluis Blancafort

Institut de Quimica Computacional i Catalisi (IQCC) i Departament de Quimica, Facultat de Ciencies

Universitat de Girona

C/M. A. Capmany 69, 17003 Girona (Spain)

E-mail: 1 luis.blancafort@udg.edu

\begin{abstract}
Electronic conjugation through covalent bonds is generally considered as the basis for the electronic transition of organic luminescent materials. Tetraphenylethylene (TPE), an efficient fluorophore with aggregation-induced emission (AIE) character, its blue photoluminescence in aggregate state is always ascribed to the through-bond conjugation (TBC) among the four phenyl rings and the central $\mathrm{C}=\mathrm{C}$ bond. Herein, systematic spectrometry studies and ab initio theoretical simulation were conducted for TPE and its derivatives, and intramolecular through-space interaction (TSI) between two vicinal phenyl rings is proved as the origin of the blue emission. Furthermore, aided by the evaluation of excitedstate decay dynamics, the non-luminescent nature of TPE in solution is revealed as the result of excited-state evolution towards conical intersections via isomerization and cyclization. In aggregate state, the excited-state TSI (ESTSI) is stabilized by the restriction of intramolecular motions, and strong blue emission from through-space conjugation is induced. The mechanistic model of ESTSI delineated in this work provides a new strategy to design luminescent materials beyond the traditional theory of $\mathrm{TBC}$, and expands the quantum understanding of molecular behavior into the aggregate level.
\end{abstract}

\section{Introduction}

Molecular structures and properties govern the macroscopic performance of the materials according to the reductionism conjecture in molecular science. ${ }^{[1]}$ In the field of organic photoelectronics, the optical performances of luminescent materials are mainly manipulated by modifying the electronic conjugation through chemical bonds at the single-molecule level. ${ }^{[2]}$ Therein, the strongest covalent bonds establish the molecular frameworks for most organic luminogenic molecules (luminogens), upon which the $\pi$ electrons and lone pairs of electrons can migrate or be delocalized to form the conjugated structures. ${ }^{[3]}$ Consequently, the electronic transitions involved in the optical absorption and emission arise on such structures with through-bond conjugation (TBC). It is well known that larger TBC will generate more probable electronic transition and better photophysical performance. ${ }^{[4]}$ Additionally, emission colors covering ultraviolet to near-infrared regions can be achieved by tuning the $\pi$ conjugation. ${ }^{[4]}$ With such guidance of TBC at the molecular level, all kinds of luminescent molecules with versatile functionalities have been created, which show excellent optical performance in the isolated state or dilute solution. Nevertheless, traditional chromophores with extended TBC structures usually suffer the emission quenching in their concentrated solution or solid state, which definitely hampers practical applications in the solid state and also invalidates the principles for molecular design based on the TBC. ${ }^{[5]}$

From the viewpoint of molecular photophysics, it is anticipated that molecules or subunits without considerable TBC can hardly afford strong light emission in the isolated state due to their neglectable transition dipole moment and the overlarge energy gaps. Indeed, taking the proteins and cellulose as examples, they possess only isolated amino, carbonyl or hydroxyl groups in their molecular backbones, so their dilute solutions are nonemissive upon UV excitation. Interestingly, once the aggregation happens or in the solid state, they can emit visible light and show the marvelous clusterization-triggered emission (CTE) phenomenon. ${ }^{[6]}$ What is the origin of such unusual photoluminescence (PL)? Which interaction promotes the electronic transition as there is nearly no TBC along the molecular backbones of the protein and cellulose?

Thanks to the enthusiastic research efforts, it is revealed that through-space interaction (TSI) among the nonconjugated subunits, such as isolated aromatic rings and heteroatoms with lone-pair electrons, plays an essential role in CTE, as the formed TSI will generate orbital splitting and coupling after clusterization and then decrease the energy level for visible light emission. ${ }^{[6]}$ Whereas the effect of TSI on luminescence is generally neglected in the traditional photophysical studies because the dilute solution is usually applied as the ideal situation, in which the vigorous 
molecular motions will destabilize the TSI. When the molecular species work in the aggregate or solid state, the influence from TSI may be dramatically different, especially for the nonconjugated systems. Currently, the TSI model has been successfully applied to explain the CTE in nonconjugated polymers and small molecules, such as bovine serum albumin and tetraphenylethane. ${ }^{[7]}$ Thereinto, it has been found that the excitedstate aromatic interaction can endow the red-shifted emission in molecular rotors. ${ }^{[8]}$ The circularly delocalized $\pi$-electron density has been coined to describe the TSI in the tetrafuranethylene. ${ }^{[9]}$ Furthermore, the through-space charge transfer has been revealed to facilitate the thermally activated delayed fluorescence. ${ }^{[10]}$ However, it remains a black box that what the photophysical nature of TSI is, where TSI happens and how TSI takes a specific effect on the photon absorption and emission. The deeper comprehension and description from quantum mechanics of TSI are highly desired and there is plenty of space for exploring the power of TSI in photophysical processes.

In this work, we took the tetraphenylethylene (TPE) containing four phenyl rings with steric proximity as the model compound to draw a clear mechanistic picture of TSI in the excited-state photophysical processes. TPE shows fantastic AIE behavior: it only emits weak ultraviolet light in dilute solution, but much enhanced visible blue light appears in its aggregate state. However, such dramatic variation of luminescent wavelength in this simple and well-known conjugated system has not been rigorously studied. It remains controversial and confusing how the visible emission of TPE is generated in the aggregate state since the excited-state energy level from TBC does not match with the blue emission. Does it come from TSI? Where and how is the underneath interaction involved? Through systematic spectrometry studies and ab initio theoretical simulation of TPE and its derivatives, we have confirmed the predominant effect of TSI in the blue emission of TPE and finally concluded a clear model for the formation and diminishment of TSI in the isolated and aggregate states, respectively. The investigation of TSI will undoubtedly contribute to revealing the working mechanisms of molecular rotors with separated aromatic rings, and fertilize the photophysical theories. The synergistic effect of TSI and TBC on the luminescence in the aggregate state will provide novel strategies to design efficient and versatile chromophores, and expand the quantum understanding of molecular behavior into the aggregate level. ${ }^{[1]}$

\section{Results and Discussion}

Molecular photophysics has demonstrated that larger molecular through-bond conjugation always furnishes longer wavelengths of photon absorption $\left(\lambda_{\mathrm{abs}}\right)$ and emission $\left(\lambda_{\mathrm{em}}\right)$, as exemplified by the classical aromatic systems in Figure 1a. The benzene (BZ) ring serves as the fundamental conjugation element and shows $\lambda_{\mathrm{abs}}$ and $\lambda_{\mathrm{em}}$ shorter than $300 \mathrm{~nm}$ in the dilute solution. ${ }^{[12]}$ With progressive fusion of benzene rings, the $\lambda_{\mathrm{abs}}$ increases from $268 \mathrm{~nm}$ in BZ to $286 \mathrm{~nm}$ in naphthalene (NA) and then $377 \mathrm{~nm}$ in anthracene (AN). ${ }^{[12]}$ Similar shifting trend appears in their $\lambda$ em. Comparing to the tetraphenylethane $\left(s\right.$-TPE) with weaker TBC $\left(\lambda_{\mathrm{abs}}=270 \mathrm{~nm}, \lambda_{\mathrm{em}}=290 \mathrm{~nm}\right)$, both the absorption and emission of TPE are redshifted to 307 and $375 \mathrm{~nm}$, respectively, which yet remain in the ultraviolet region. Despite their difference in TBC, however, a dramatic bathochromic shift arises in the emission of both TPE and $s$-TPE in the solid state comparing to their dilute solution (Figure 1b) with large stocks shifts of $133 \mathrm{~nm}$ for TPE and $190 \mathrm{~nm}$ for $s$-TPE, respectively, indicating an unknown excited-state interaction beyond the TBC is involved in the solid state of TPE and $s$-TPE.

To further explore this exotic phenomenon in the solid state, we measured the PL spectra of $s$-TPE ${ }^{[7 b]}$ and TPE in THF/water mixture with different water fractions $\left(f_{\mathrm{w}}\right)$ to kinetically monitor the emission variation within the process of aggregation. As shown in Figure 2, both $s$-TPE and TPE show ultraviolet emission in pure THF solution. With the increase of $f_{\mathrm{w}}$, molecular aggregates are gradually formed and emission peaks at around $460 \mathrm{~nm}$ arise with the $f_{\mathrm{w}}$ above $70 \%$ for $s$-TPE, which are intensified with further increasing the $f_{\mathrm{w}}$ (Figure $2 \mathrm{a}$ ).
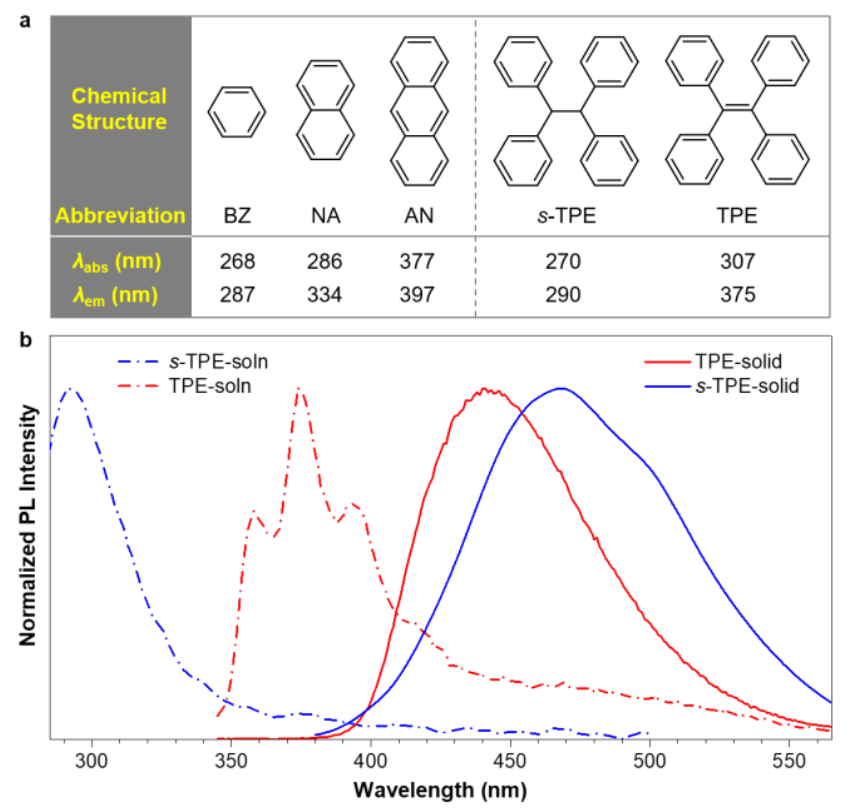

Figure 1. (a) Chemical structures, absorption $\left(\lambda_{\mathrm{abs}}\right)$ and emission wavelengths $\left(\lambda_{\mathrm{em}}\right)$ in the isolated state of benzene (BZ), naphthalene (NA), anthracene (AN), tetraphenylethane (s-TPE) and tetraphenylethylene (TPE). (b) Normalized photoluminescence (PL) spectra of TPE and $s$-TPE in solution (soln) and solid (crystalline) states. $[s-\mathrm{TPE}]=10^{-4} \mathrm{M},[\mathrm{TPE}]=10^{-5} \mathrm{M} . \lambda_{\mathrm{ex}}(s-\mathrm{TPE})=280 \mathrm{~nm}, \lambda_{\mathrm{ex}}(\mathrm{TPE})=307 \mathrm{~nm}$. 
TPE exhibits the same luminescent behaviors but its emission is induced at $80 \%$ due to the lower concentration than $s$-TPE. The intermolecular interaction such as $\pi-\pi$ stacking might be considered as a reason for the red-shift of emission in the aggregate state. Therefore, the crystal packing obtained by the X-ray diffraction technique was analyzed for both $s-\mathrm{TPE}^{[7 \mathrm{~b}]}$ and $\mathrm{TPE}^{[13]}$ (Figure $2 \mathrm{c}$ and $2 \mathrm{~d}$ ). Nevertheless, it is found that the closest intermolecular distance between the adjacent phenyl rings of $s$-TPE and TPE are $4.409 \AA$ and $4.926 \AA$, respectively, which are far beyond the sum of the Van der Waals radius of the carbon atom (3.4 $\AA$ ), suggesting the negligible intermolecular $\pi-\pi$ interaction in their aggregates. Thus, if the intermolecular interaction takes no effect on the dramatic bathochromic shift of $\lambda_{\mathrm{em}}$, the intramolecular interaction should have made a major contribution. It is noteworthy that TPE shares similar luminescent features with $s$-TPE which consists of four isolated phenyl rings without the TBC. Our previous work has revealed that the TSI in $s$-TPE plays a vital role in its abnormal emission at $460 \mathrm{~nm}$. Thereupon, it is anticipated that the intramolecular interaction among four phenyl rings of TPE may play a considerable part in its photophysical properties.

To investigate the intramolecular interactions among four phenyl rings of TPE and their functions on the electronic transition, PL spectra of TPE and three phenylethylene derivatives were measured in the dilute solution at room temperature (RT) and cryogenic temperature (LT), respectively (Figure 3). The number of phenyl rings is progressively decreased from TPE to diphenylethylene (DPE), and the two adjacent phenyl rings are switched from the vicinal position in $v$-DPE to the geminal position in $g$-DPE to mimic the inter-phenyl interaction in different parts of TPE. As shown in Figure $3 \mathrm{a}$, for TPE, its $\lambda_{\mathrm{em}}$ in the solution at LT is almost the same as that in the solid state, as compared to its solution at RT which only shows faint emission in the ultraviolet region.
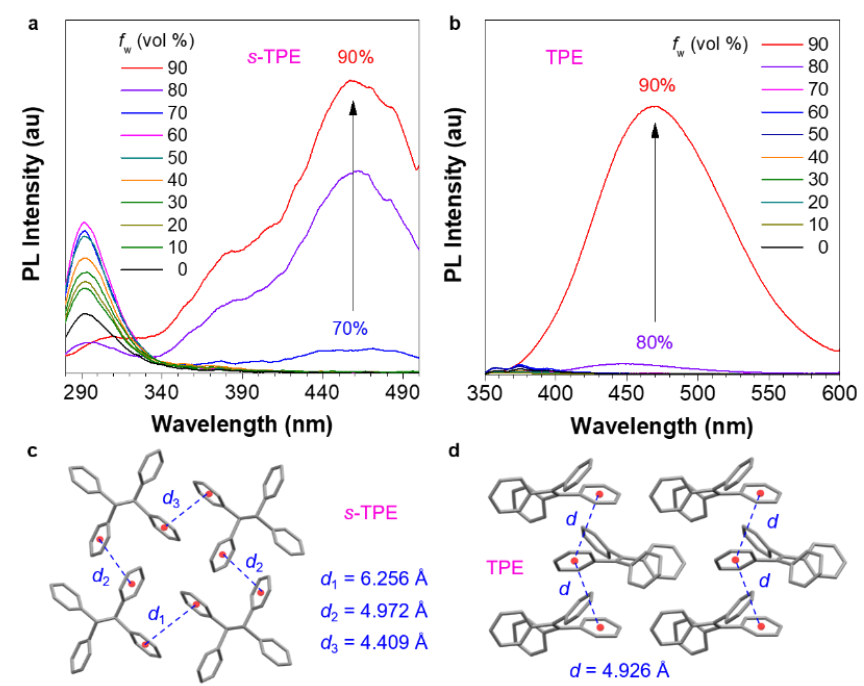

Figure 2. PL spectra of (a) $s$-TPE and (b) TPE in THF/water mixtures with different water fractions. $[s-\mathrm{TPE}]=10^{-4} \mathrm{M},[\mathrm{TPE}]=10^{-5} \mathrm{M} . \lambda_{\mathrm{ex}}(\mathrm{s}-\mathrm{TPE})=280 \mathrm{~nm}$, $\lambda_{\text {ex }}($ TPE $)=307 \mathrm{~nm}$. Molecular packing arrangements in crystals of (c) $s$-TPE and (d) TPE.

After deducting one of the phenyl rings in TPE, the obtained triphenylethylene (TriPE) surprisingly exhibits similar luminescent variation from RT to LT as TPE (Figure 3b). A drastically enhanced PL peak emerges in the visible region after cooling the dilute solution of TriPE from RT into $77 \mathrm{~K}$, despite the smaller number of phenyl rings and consequently weakened TBC. As shown in Figure $3 \mathrm{c}$ and $3 \mathrm{~d}$, one more phenyl ring was further deducted from TriPE and obtained $v$-DPE and $g$-DPE with two phenyl rings in vicinal $(v)$ and geminal $(g)$ steric proximity, respectively. The main PL peaks in the RT solution of these two compounds are located in the ultraviolet region, and these peaks remain in the LT solution with enhanced intensity. However, these two DPE isomers bear intensified shoulder peaks above $400 \mathrm{~nm}$ in LT solution, which is negligible in the RT solution. Therefore, the red-shift in PL can also happen even with only two phenyl rings on the molecular skeleton. The comparison among the four compounds leads to the conclusion that the inter-phenyl interaction contributes predominantly to the bathochromic shift of the PL of TPE from solution to aggregate state, namely, the through-space interaction (TSI). But it remains unclear where and how the TSI forms.

To answer these two important questions, the ab initio theoretical calculation was conducted to explore the origin of the inter-phenyl TSI in TPE. The Atoms-in-Molecules (AIM) analysis using the Mulfiwfn 3.7 package ${ }^{[14]}$ was employed for $v$-DPE and $g$-DPE which are the basic structural elements of the TPE molecule, aiming to visualize the interaction paths and locate the key conformations possessing the TSI. AIM analysis is a powerful quantum method to analyze the topology of electron density, thereinto, the bond and cage critical points indicate the extreme points of electron density on the bond paths and enclosed space formed by rings, respectively. ${ }^{[15]}$ 

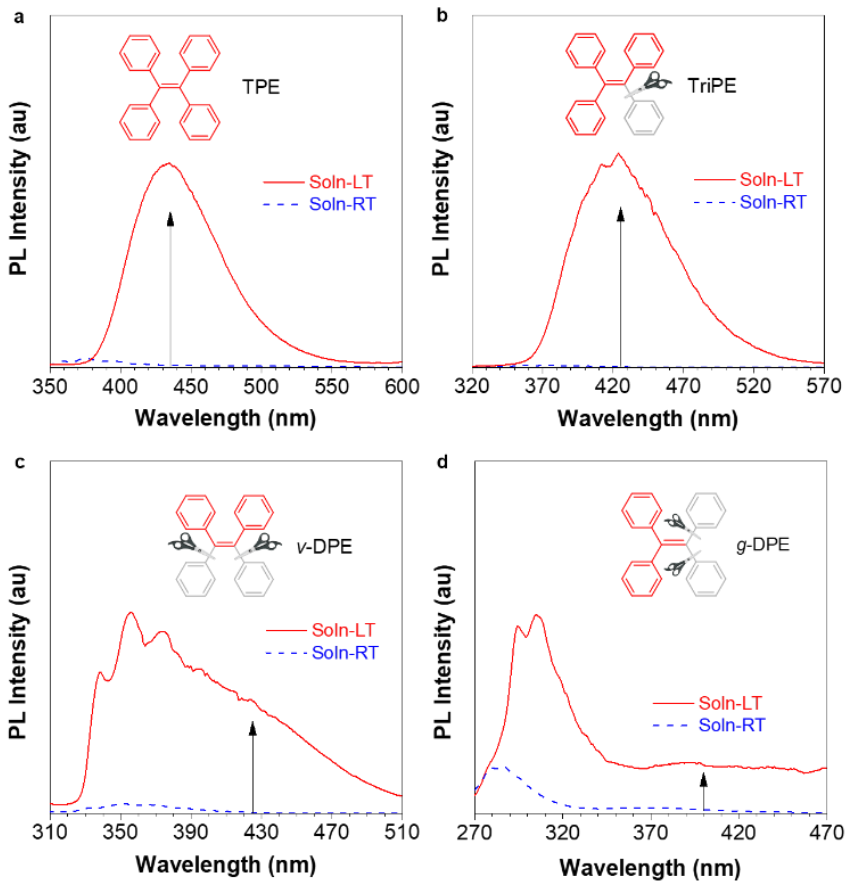

Figure 3. PL spectra of (a) tetraphenylethylene (TPE), (b) triphenylethylene (TriPE), (c) (Z)-1,2-diphenylethylene ( $v$-DPE) and (d) 1,1-diphenylethylene ( $g$-DPE) in THF solutions at $298 \mathrm{~K}(\mathrm{RT})$ and $77 \mathrm{~K}(\mathrm{LT})$. [TPE $]=[\mathrm{TriPE}]=[v$-DPE $]=[g-\mathrm{DPE}]=10^{-5} \mathrm{M} . \lambda_{\mathrm{ex}}(\mathrm{TPE})=307 \mathrm{~nm}, \lambda_{\mathrm{ex}}($ TriPE $)=300 \mathrm{~nm}, \lambda_{\mathrm{ex}}(v-\mathrm{DPE})=274 \mathrm{~nm}$, $\lambda_{\mathrm{ex}}(g-\mathrm{DPE})=250 \mathrm{~nm}$

Through AIM analysis, a clear mapping of the intramolecular through-space electronic interaction among the subunits can be depicted. The potential energy surfaces were firstly scanned with rotation of vicinal phenyl rings (dihedral angle $\alpha$ ) for $v$-DPE (Figure 4a) and geminal phenyl rings (dihedral angle $\gamma$ ) for $g$-DPE (Figure $4 \mathrm{c}$ ), and the twisting of $\mathrm{C}=\mathrm{C}$ double bond (dihedral angle $\beta$ ), to simulate the dominant vibrational relaxation within the excited-state decay process (see calculation details in the Supporting Information). Each conformation was optimized by fixing the corresponding values of torsion and twisting angles. Afterwards, the AIM analysis was applied on the optimized structures, and bond/cage critical points were plotted between the phenyl rings. As shown in Figure 4a, the hydrogen-hydrogen interaction has been found between the hydrogen atoms attached to the C1 and C6 in $v$-DPE with both $\alpha$ and $\beta$ smaller than $40^{\circ}$. While for $g$-DPE, the hydrogen-hydrogen interaction appears in the conformations with $\gamma$ smaller than $30^{\circ}$ (Figure 4c). Furthermore, with appropriate twisting angle $\left(\beta=0-40^{\circ}\right)$ and torsion angles $(\alpha=$ $10-40^{\circ}$ for $v$-DPE, $\gamma=30^{\circ}$ for $g$-DPE), the conformations of both DPE isomers in the area bounded by magenta lines show multiple bonding paths, i.e., hydrogen-hydrogen, hydrogen-carbon, and carbon-carbon bonding, demonstrating unambiguous through-space interaction between the phenyl rings in both vicinal and geminal positions. Meanwhile, the concentrated electron density on these bonding paths will further facilitate the throughspace $\pi$-electron delocalization, and then promote the electronic transitions in the luminescent processes. ${ }^{[16]}$ Multiple through-space interactions constitute virtual 6- or 7-membered rings on the backbones of both DPE isomers (Figure 4b, 4d). Meanwhile, multiple ring planes intersecting with each other form the enclosed electron cage as indicated by the cage critical point, which will contribute to the stabilization of the whole system. ${ }^{[17]}$ The vicinal and geminal TSI have been visualized by AIM analysis for the subparts of TPE, and the essential structural parameters required for efficient TSI have also been confirmed. But why is TPE non-luminescent in the RT solution? Why does TSI not take effect in the isolated TPE molecule? According to the experimental observation of enhanced visible emission in the solid state and LT solution, it is anticipated that the active intramolecular motions and corresponding vibronic coupling in RT solution may hamper TSI. ${ }^{[18]}$

Therefore, the two-dimensional potential energy surfaces in both ground and excited states were calculated to monitor the kinetics of TSI in TPE with the torsion of phenyl rings and the twisting of the $\mathrm{C}=\mathrm{C}$ double bond in both THF solution and crystalline state (see calculation details in the Supporting Information). As shown in Figure S1b and S1c, upon excitation from the local minimum of ground state to its Frank-Condon point, TPE undergoes the barrierless vibrational relaxation with the rotation of phenyl rings and the twisting of central $\mathrm{C}=\mathrm{C}$ bond, which provides appropriate torsion angles and inter-phenyl C-C distances for the formation of efficient TSI. Whereas the drastic torsion of phenyl rings and twisting of the $\mathrm{C}=\mathrm{C}$ bond may lead to energy crossing areas associated with the photo-induced cyclization and $\mathrm{E} / \mathrm{Z}$ isomerization for the ultrafast nonradiative decay ${ }^{[19]}$ On the contrary, the PES in the crystalline state (Figure S1e and S1f) shows huge barriers for the phenyl-ring torsion and double-bond twisting, which constrains the conformational variation within a narrow area on the PES and thus stabilizes the emissive conformations. To decipher the intramolecular interactions involved in TPE, the conformations of the conical intersection (CI) points for both cyclization $\left(\mathrm{CI}_{\mathrm{c}}\right)$ and isomerization $\left(\mathrm{CI}_{\mathrm{i}}\right)$ were calculated. The minimum energy path (MEP) for these two dominant excited-state decay channels $\left(\mathrm{CI}_{\mathrm{c}}\right.$ and $\left.\mathrm{CI}_{\mathrm{i}}\right)$ were further simulated in THF solution using the intrinsic reaction coordinates (IRC) method to monitor the excited-state structural evolution of TPE in real time. Herein, the AIM analysis was applied on the key conformations extracted from the IRC pathways in solution (Figure 5a, left) and crystalline (Figure 5a, right) states. The relevant key structural parameters are summarized in Figure S2. 


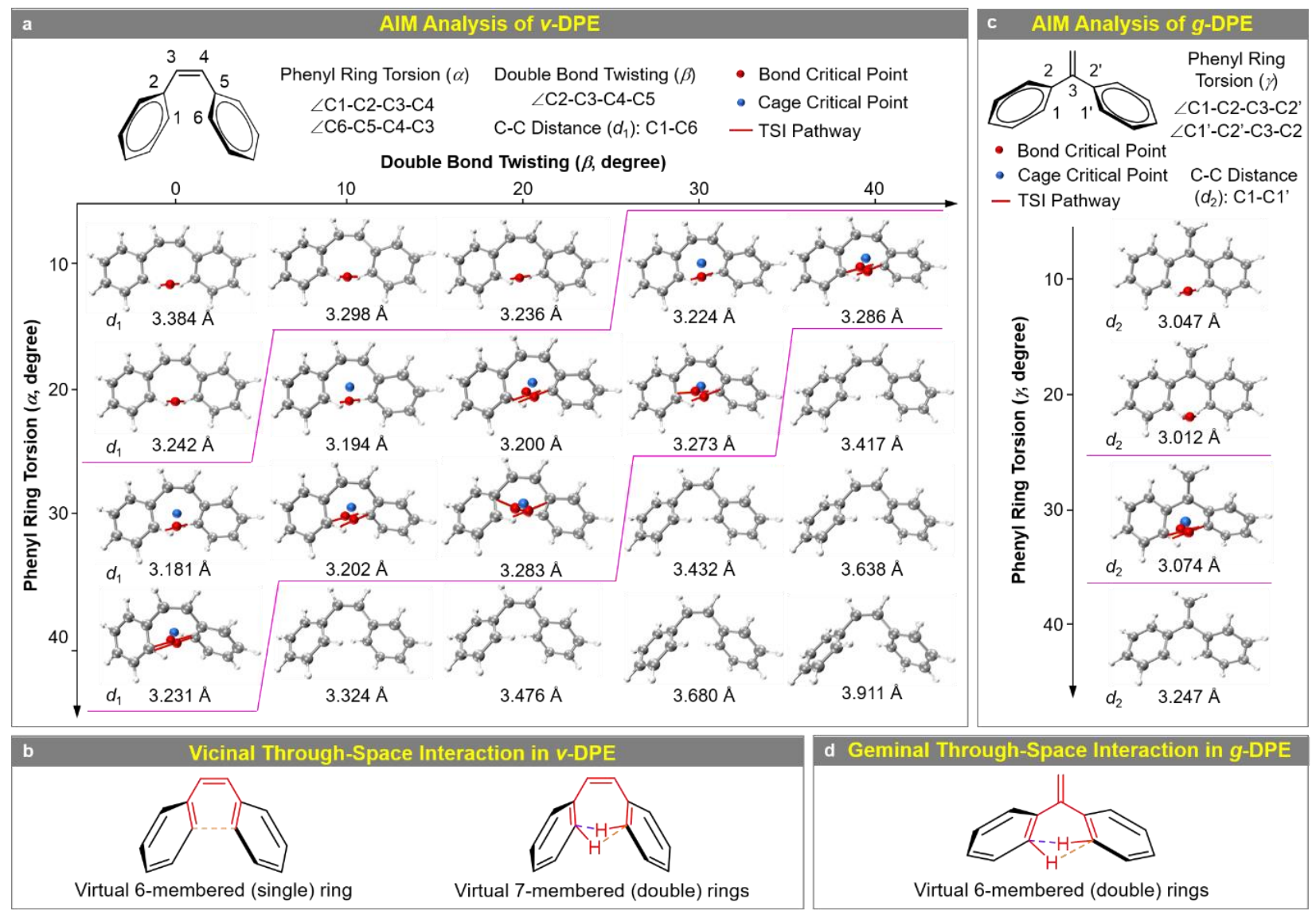

Figure 4. (a) Atom-in-molecules (AIM) analysis of selected conformations of (Z)-diphenylethylene ( $v$-DPE) from the relaxed scans of potential energy surfaces with the torsion of phenyl rings $(\alpha)$ and the twisting of $\mathrm{C}=\mathrm{C}$ double bond $(\beta)$. Conformations of $v$-DPE with stronger through-space interaction (TSI) are separated by the magenta lines. TSI pathways between the vicinal phenyl rings are highlighted by red lines. (b) Vicinal TSI in $v$-DPE with intramolecular interactions highlighted by orange and violet lines. (c) AIM analysis of selected conformations of 1,1-diphenylethylene ( $g$-DPE) from the relaxed scans of potential energy surfaces with the torsion of the phenyl rings $(\gamma)$. TSI pathways between the geminal phenyl rings are highlighted by red lines. (d) Geminal TSI in $g$-DPE with the intramolecular interactions highlighted by orange and violet line

Figure 5a indicates that the $\mathrm{FC}^{*}$ soln structure exhibits no bonding path and bond/cage critical points, revealing the absence of TSI for this conformation with four identical phenyl-ring torsion angles $(\theta)$ of $52^{\circ}$ and $\mathrm{C}=\mathrm{C}$ bond twisting angle $(\varphi)$ of $10^{\circ}$ in solution. Starting from the $\mathrm{FC} *$ soln point with energy of $4.19 \mathrm{eV}$, TPE firstly attains a bifurcation point (Bif) with a rapid drop in energy $(-0.93 \mathrm{eV})$ but slight increase of $\varphi\left(+12^{\circ}\right)$, decrease of $\theta\left(-20^{\circ}\right)$ and shortening of the vicinal C-C distance $\left(d_{1}\right)$ associated with the photo-induced cyclization $(-0.4 \AA)$ as shown in Figure S2. The approaching of two pairs of vicinal phenyl rings in the Bif structure results in the prominent TSI between them as indicated by the bond paths and bond critical points (Figure 5a). Further vibrational relaxation of Bif structure leads to the $\mathrm{CI}_{\mathrm{c}}$ point with decreasing $d_{1}$ and $\mathrm{CI}_{\mathrm{i}}$ point with increasing $d_{1}$, respectively. From Bif to $\mathrm{CI}_{\mathrm{c}}$ point, one pair of vicinal phenyl rings further approaches to each other but the other pair separates away, which enhances the vicinal TSI between the corresponding phenyl rings as indicated by the bond paths at the transition $\left(\mathrm{T}_{\mathfrak{c}}\right)$ and $\mathrm{CI}_{\mathfrak{c}}$ points. On the other pathway towards the $\mathrm{CI}_{i}$ point for isomerization, the $\varphi$ drastically increases from $10^{\circ}$ to $85^{\circ}$ with the planarization of two pairs of geminal phenyl rings from $52^{\circ}$ to $16^{\circ}$, which largely increases the distances between vicinal phenyl rings. Hence, the points of $\mathrm{T}_{\mathrm{i}}, \mathrm{S}_{1, \mathrm{~min}, \mathrm{soln}}$ and $\mathrm{CI}_{\mathrm{i}}$ mainly show the through-space hydrogen-hydrogen interaction between the geminal phenyl rings. From the AIM analysis, it is found that TPE tends to form the TSI between the vicinal phenyl rings and/or between geminal phenyl rings during the excited-state evolution in THF solution. However, it will suffer ultrafast nonradiative decay to the ground state once the conformation reaches CI points. While for the crystalline state, the FC* ${ }_{\text {xtal }}$ structure generates TSI between two vicinal phenyl rings.

When it relaxes to the $\mathrm{S}_{1, \mathrm{~min} \text {,xtal }}$ point, this conformation shows stronger TSI with both hydrogen-carbon and carbon-carbon interaction. Furthermore, as shown in Figure 5b, the frontier molecular orbitals at the minima of $S_{0}$ and $S_{1}$ states exhibit evident orbital overlap in the lowest unoccupied molecular orbital (LUMO) at $\mathrm{S}_{1, \mathrm{~min}, \mathrm{xtal}}$, indicating that the TSI between vicinal phenyl rings of TPE in the aggregate state can efficiently promote the electronic delocalization and generate the bathochromic luminescence. The weak interactions in TPE were also investigated using the reduced density gradient (RDG) and electrostatic potential (ESP) mapping for both the $\mathrm{S}_{1, \mathrm{~min}, \mathrm{soln}}$ and $\mathrm{S}_{1, \mathrm{~min}, \mathrm{xtal}}$ points (Figure $\mathrm{S} 3$ ).

It shows that evident Van der Waals interactions emerge between the adjacent phenyl rings in both structures, and the electrostatic attraction is formed between the hydrogen and carbon atoms with positive and negative ESP, respectively. Therefore, in Figure S3c, it is proposed that the Van der Waals and electrostatic interactions between the neutral or partially charged phenyl rings ${ }^{[20]}$ can drive the formation of TSI. To gain deeper insights into the kinetic evolution of TSI in TPE, the potential energy surface (PES) along the dominant decay pathways was evaluated. The schematic illustration of calculated MEP shown in Figure 5c reveals that, in THF solution, the excited-state conformation will decay along the 
twisting of the $\mathrm{C}=\mathrm{C}$ bond and planarization of four phenyl rings (Figure S2) towards the $\mathrm{CI}_{\mathrm{i}}$ point. The decay pathway of $\mathrm{Bif}_{\mathrm{f}}$ to $\mathrm{CI} \mathrm{I}_{\mathrm{c}}$ points is also achievable. Hence, it reveals that the formed TSI in THF solution will be broken by the excited-state intramolecular motions, resulting in the severe nonradiative decay through the conical intersection to the ground state. In comparison, much higher energy barrier for structural evolution is needed in the aggregate state than that in THF solution, which restricts the intramolecular motion and stabilizes the conformation with efficient TSI (Figure 5d). Finally, the restriction of intramolecular motions dramatically blocks the nonradiative decay and boosts the visible TSI luminescence of TPE in the aggregate state. Furthermore, the relatively looser intermolecular packing in the amorphous state than in the crystalline state will provide more active molecular motions, which leads to the less steep PES and more bathochromic emission than the crystal state as illustrated in Figure 5d.
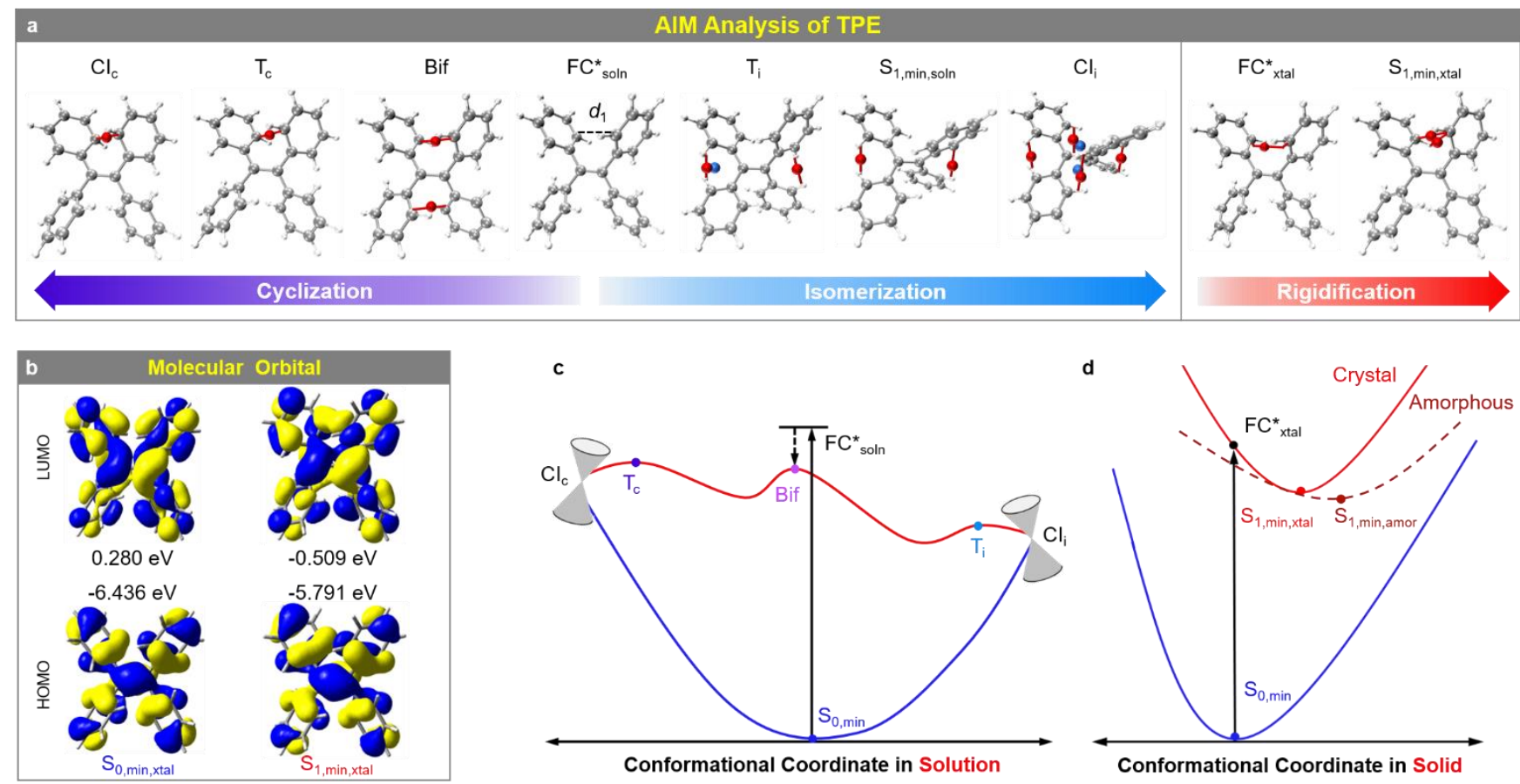

C

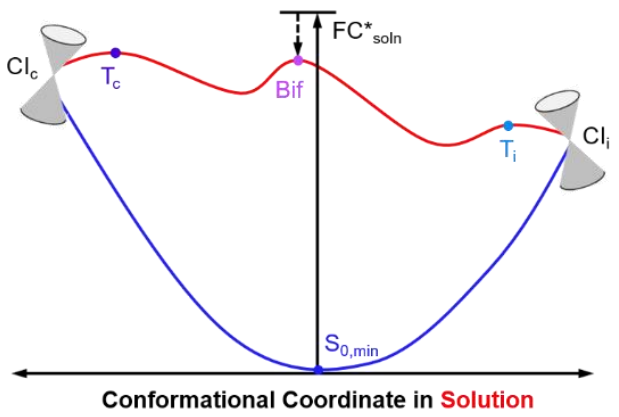

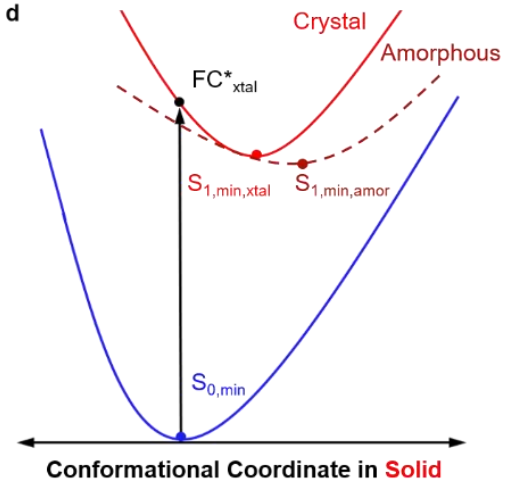

Figure 5. (a) Atom-in-molecules (AIM) analysis of key conformations extracted from the IRC pathways in the excited state in solution and crystal. (b) Molecular orbitals associated with the electronic transitions at the minima in the $S_{0}$ state and $S_{1}$ state. (c) Schematic illustration of IRC pathways calculated in the $S_{1}$ state of TPE in THF solution from the Frank-Condon point (FC*) to the bifucation point (Bif) and then to the conical intersections of E/Z isomerization (CI $\mathrm{F}_{\mathrm{i}}$ and cyclization $\left(\mathrm{CI}_{\mathrm{c}}\right) . \mathrm{T}_{\mathrm{c}}=$ transition state along the cyclization path, $\mathrm{T}_{\mathrm{i}}=$ transition state along the E/Z isomerization path. (d) Schematic illustration of potential energy profiles in the $S_{0}$ and the $S_{1}$ states of TPE in crystalline and amorphous states.

In conclusion, as shown in Figure 6a, TPE exhibits weak ultraviolet light emission from TBC in dilute solution due to the active intramolecular twisting and torsion, which lead to the ultrafast nonradiative decay of excitons with TSI through two conical intersection points. However, when it aggregates into the solid state, the conformations with the excited-state through-space interactions (ESTSI) in the vicinal or the geminal position can be effectively stabilized due to the strong steric hindrance in the aggregate environment (Figure 6b) and the steric antagonism between the vicinal pair and the geminal pair of phenyl rings. Consequently, the stabilized electron delocalization and enhanced through-space electronic conjugation dramatically boost the blue emission of TPE in the aggregate state. The ESTSI widely exists in organic luminescent materials. Taking the compounds with multiple phenyl rings as examples (Figure 6c), when the number of carbon atoms between the phenyl rings (n) equals to 1, it furnishes the diphenylmethane (DPM) with isolated $\pi$ subunits. DPM has been revealed with geminal TSI which is also termed as homoconjugation. ${ }^{[21]}$

As a result, the ESTSI in DPM leads to strong visible light in the aggregate state. When $\mathrm{n}$ increases to 2, the scenario will be related to the vicinal ESTSI as discussed in this work. When there are 3 carbon atoms inserted between two phenyl rings, the phenyl rings can arrange in a face-to-face pattern similar to the repeating unit of polystyrene and form parallel ESTSI, as evidenced by the blue PL in syndiotactic polystyrene. ${ }^{[22]}$ As shown in Figure 6d-g, comparing to the classical twisted intramolecular charge transfer (TICT) and excited-state intramolecular proton transfer (ESIPT), ESTSI is formed on the relatively planar PES in the isolated state but can be finally stabilized and enhanced in the aggregate state. It can facilitate the through-space electron delocalization and promote the bathochromic light emission with increased efficiency. The TICT effect can also realize the red-shifted emission but with decreased efficiency, which mainly depends on the charge transfer from the electron donor to acceptor through the twisted covalent bond. ${ }^{[23]}$ However, the aggregation is unfavorable for TICT due to the restricted twisting. For ESIPT, the tautomerization between the enol and keto structures through the hydrogen bonding will lead to red-shifted PL but the emission efficiency cannot be precisely manipulated in varied conditions. ${ }^{[24]}$ The chemical processes related to ESIPT will complicate the molecular design and application scenarios. 


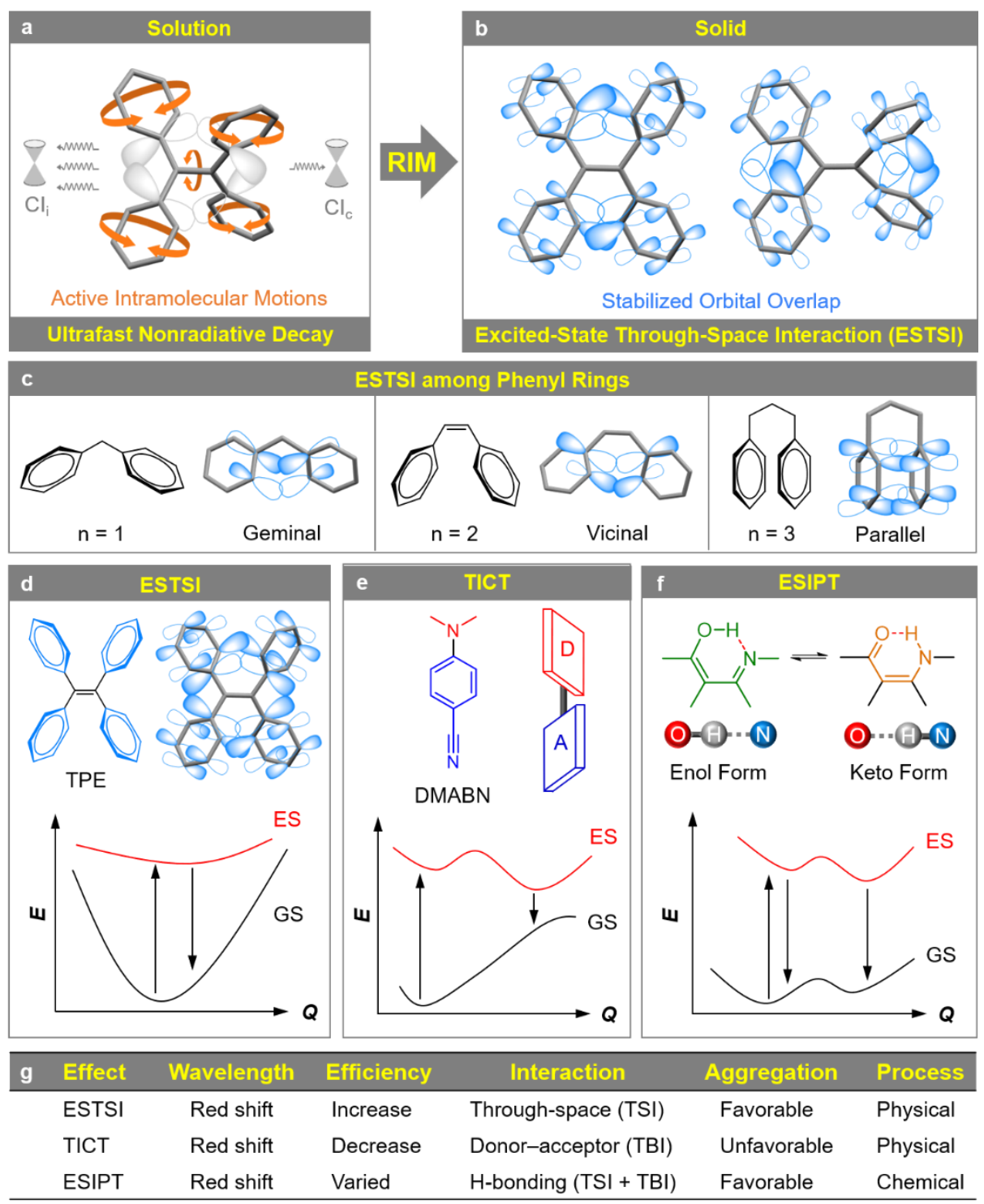

Figure 6. Schematic illustrations of (a) excited-state decay processes of TPE caused by the active intramolecular motions in the solution state, (b) excited-state through-space interaction (ESTSI) stabilized by the restriction of intramolecular motions (RIM) in the solid state and $(\mathrm{c})$ ESTSI systems with $\mathrm{n}=1,2,3$. CI $\mathrm{i}_{\mathrm{i}}=\mathrm{conica}$ intersection of $\mathrm{E} / \mathrm{Z}$ isomerization, $\mathrm{CI}_{\mathrm{c}}=$ conical intersection of cyclization, $\mathrm{n}=$ number of carbon atom(s) between the two phenyl rings with through-space interaction. Comparison between working mechanisms of (d) ESTSI, (e) twisted intramolecular charge transfer (TICT) and (f) excited-state intramolecular proton transfer (ESIPT) systems. (g) Characteristics of ESTSI, TICT and ESIPT effects.

\section{Conclusion}

In this work, we systematically investigated the photophysical properties of TPE and its derivatives through spectrometry and ab initio theoretical simulation. It reveals that the enhanced blue emission of TPE in the aggregate state originates from the excited-state through-space interaction but not the through-bond conjugation among four phenyl rings and the central $\mathrm{C}=\mathrm{C}$ bond. In dilute solution, the ESTSI effect will be diminished by drastic molecular motions, resulting in the non-luminescent feature. The restriction of intramolecular motions in the cryptogenic condition or the aggregate state blocks the nonradiative decay pathways and stabilizes the vicinal TSI conformations. Hence, the stabilized ESTSI generates expanded through-space electron delocalization and promotes the visible emission in the solid state. The ESTSI provides a new platform to deal with electronic properties beyond the traditional theories based on through-bond conjugation. It is anticipated that ESTSI will become a significant photophysical mechanism for both the academic research and applications of smart luminescent materials with multi-dimensional interactions.

\section{Acknowledgements}

We are grateful for financial support from the National Science Foundation of China (21788102 and 81501591 ), the Research Grants of Council of Hong Kong (16305518, 16307020, 16306620, N-HKUST609/19, and C6014-20W), and the Innovation of Technology Commission (ITC- 
CNERC14SC01). H. Z thanks the support from the Fundamental Research Funds for the Central Universities and the Open Fund of Guangdong Provincial Key Laboratory of Luminescence from Molecular Aggregates, and South China University of Technology (2019B030301003);

\section{Conflict of interest}

The authors declare no conflict of interest.

Keywords: Through-space Interaction • Aggregation-induced emission • Atom in Molecules • Conical Intersection• Excited-state Decay Dynamics

[1] a) A. Jablonski, Nature 1933, 131, 839-840; b) J. Weiss, Nature 1943, 152, 176-178; c) W. T. Astbury, Nature 1961, 190, $1124-1125$.

[2] a) L. Zhan, Z. Chen, S. Gong, Y. Xiang, F. Ni, X. Zeng, G. Xie, C. Yang, Angew. Chem. Int. Ed. 2019, 58, 17651-17655; b) M. Y. Wong, E. Colman, Adv. Mater. 2017, 29, 1605444; c) P. Data, Y. Takeda, Chem. Asian J. 2019, 14, 1613-1636.

[3] a) J. Thiele, Liebigs Ann. Chem. 1899, 306, 87-142; b) C. A. Coulson, H. C. Longuet-Higgins, Proc R Soc Lond Ser A 1947, 191, 39-60. c) H. Hosoya, Bull. Chem. Soc. Jpn. 2019, 92, 205-215.

[4] a) O. Ostroverkhova, Chem. Rev. 2016, 116, 13279-13412; b) W. Wan, D. Tian, Y. Jing, X. Zhang, W. Wu, H. Ren, H. Bao, Angew. Chem. Int. Ed. 2018, 57,15510-15516; c) J. Li, A. Terec, Y. Wang, H. Joshi, Y. Lu, H. Sun, M. C. Stuparu, J. Am. Chem. Soc. 2017, 139, 3089-3094.

[5] J. Mei, N. L. C. Leung, R. T. K. Kwok, J. W. Y. Lam, B. Z. Tang, Chem. Rev. 2015, 115, 11718-11940.

[6] a) H. Zhang, Z. Zhao, P. R. McGonigal, R. Ye, S. Liu, J. W.Y. Lam, R. T. K. Kwok, W. Z. Yuan, J. Xie, A. L. Rogach, B. Z. Tang, Mater. Today 2020, 32, 275-292; b) H. Zhang, Z. Zhao, A. T. Turley, L. Wang, P. R. McGonigal, Y. Tu, Y. Li, Z. Wang, R. T. K. Kwok, J. W. Y. Lam, B. Z. Tang, Adv. Mater. 2020, 32, 2001457; c) Z. Zhao, H. Zhang, J. W. Y. Lam, B. Z. Tang, Angew. Chem. Int. Ed. 2020, 59, 9888-9907; d) W. Li, M. Xu, C. Ma, Y. Liu, J. Zhou, Z, Chen, Y. Wang, H. Yu, J. Li, S. Liu, ACS Appl. Mater. Interfaces 2019, 11, 23512-23519; e) J. Yang, M. Fang, Z. Li, Aggregate 2020, 1, 6-18; f) Z. He, C. Ke, B. Z. Tang, ACS Omega 2018, 3, 3267-3277.

[7] a) Y. Gong, Y. Tan, H. Li, Y. Zhang, W. Yuan, Y. Zhang, J. Sun, B. Z. Tang, Sci. China: Chem. 2013, 56, 1183-1186; b) H. Zhang, X. Zheng, N. Xie, Z. He, J. Liu, N. L. C. Leung, Y. Niu, X. Huang, K. S. Wong, R. T. K. Kwok, H. H. Y. Sung, I. D. Williams, A. Qin, J. W. Y. Lam, B. Z. Tang, J. Am. Chem. Soc. 2017, 139, 16264-16272; c) H. Zhang, L. Du, L. Wang, J. Liu, Q. Wan, R. T. K. Kwok, J. W. Y. Lam, D. L. Phillips, B. Z. Tang, J. Phys. Chem. Lett. 2019, 10, 7077-7085.

[8] J. Sturala, M. K. Etherington, A. N. Bismillah, H. F. Higginbotham, W. Trewby, J. A. Aguilar, E. H. C. Bromley, A. J. Avestro, A. P. Monkman, P. R. McGonigal, J. Am. Chem. Soc. 2017, 139, 17882-17889.

[9] L. Viglianti, N. Xie, H. H. Y. Sung, A. A. Voityuk, N. L. C. Leung, Y. Tu, C. Baldoli, I. D. Williams, R. T. K. Kwok, J. W. Y. Lam, E. Licandro, L. Blancafort, B. Z. Tang, Angew. Chem. Int. Ed. 2020, 59, 8552-8559.

[10] a) Y. K. Wang, C. Huang, H. Ye, C. Zhong, A. Khan, S. Yang, M. Fung, Z. Jiang, C. Adachi, L. Liao, Adv. Optical Mater. 2020, 8, 1901150; b) H. Tsujimoto, D. Ha, G. Markopoulos, H. S. Chae, M. A. Baldo, T. M. Swager, J. Am. Chem. Soc. 2017, 139, 4894-4900; c) K. Kawasumi, T. Wu, T. Zhu, H. S. Chae, T. V. Voorhis, M. A. Baldo, T. M. Swager, J. Am. Chem. Soc. 2015, 137, 11908-11911.

[11] a) B. Z. Tang, Aggregate 2020, 1, 4-5; b) Y. Tu, Z. Zhao, J. W. Y. Lam, B. Z. Tang, Matter 2021, 4, $338-349$.

[12] a) H. Du, R. C. A. Fuh, J. Li, L. A. Corkan, J. S. Lindsey, Photochem. Photobiol. 1998, 68, 141-142; b) J. M. Dixon, M. Taniguchi, J. S. Lindsey, Photochem. Photobiol. 2005, 81, 212-213.

[13] A. Hoekstra, A. Vos, Acta Cryst. 1975, B31, 1716-1721.

[14] T. Lu, F. Chen, J. Comput. Chem. 2012, 33, 580-592.

[15] C. F. Matta, R. J. Boyd, in The Quantum Theory of Atoms in Molecules: From Solid State to DNA and Drug Design, Chapter 1, Wiley-VCH Verlag GmbH \& Co. KGaA, Weinheim, 2007, pp. 1-34.

[16] R. Hoffmann, Acc. Chem. Res. 1971, 4, 1-9.

[17] a) C. F. Matta, J. Hernandez-Trujillo, T. Tang, R. F. W. Bader, Chem. Eur. J. 2003, 9, 1940-1951; b)

[18] a) Q. Peng, Y. Yi, Z. Shuai, J. Shao, J. Am. Chem. Soc. 2007, 129, 9333-9339; b) Z. Shuai, Q. Peng, Natl. Sci. Rev. 2017, 4, 224-239; c) Y. Tu, J. Liu, H. Zhang, Q. Peng, J. W. Y. Lam, B. Z. Tang, Angew. Chem. Int. Ed. 2019, 58,14911-14914; d) P. Wei, X. Zhang, J. Liu, G. Shan, H. Zhang, J. Qi, W. Zhao, H. H. Y. Sung, I. D. Williams, J. W. Y. Lam, B. Z. Tang, Angew. Chem. Int. Ed. 2020, 59, 9293-9298.

[19] a) Y. Cai, L. Du, K. Samedov, X. Gu, F. Qi, H. H. Y. Sung, B. O. Patrick, Z. Yan, X. Jiang, H. Zhang, J. W. Y. Lam, I. D. Williams, D. L. Phillips, A. Qin, B. Z. Tang, Chem. Sci. 2018, 9, 4662-4670; b) Y. Gao, X. Chang, X. Liu, Q. Li, G. Cui, W. Thiel, J. Phys. Chem. A 2017, 121, 2572-2579; c) H. Zhang, J. Liu, L. Du, C. Ma, N. L. C. Leung, Y. Niu, A. Qin, J. Sun, Q. Peng, H. H. Y. Sung, I. D. Williams, R. T. K. Kwok, J. W. Y. Lam, K. S. Wong, D. L. Phillips, B. Z. Tang, Mater. Chem. Front. 2019, 3, 1143-1150; d) L. Blancafort, ChemPhysChem 2014, 15, 3166-3181; e) R. Crespo-Otero, Q. Li, L. Blancafort, Chem. Asian J. 2019, 14,700-714; f) Q. Li, L. Blancafort, Chem. Commun. 2013, 49, 5966-5968; g) Q. Ou, Q. Peng, Z. Shuai, J. Phys. Chem. Lett. 2020, 11, 7790-7797.

[20] a) R. W. J. Zijlstra, P. T. van Duijnen, B. L. Feringa, T. Steffen, K. Duppen, D. A. Wiersma, J. Phys. Chem. A 1997, 101, 9828-9836; b) S. Kayal, K. Roy, S. Umapathy, J. Chem. Phys. 2018,148, 024301.

[21] a) V. I. Levshin, K. I. Mamedov, S. R. Sergienko, S. D. Pustilnikova, Russ. Chem. Bull. 1959, 8, 1514-1520; b) A. Braendle, A. Perevedentsev, N. J. Cheetham, P. N. Stavrinou, J. A. Schachner, N. C. Mösch-Zanetti, M. Niederberger, W. R. Caseri, J. Polym. Sci., Part B: Polym. Phys. 2017, 55, 707-720.

[22] a) S. S. Yanari, F. A. Bovey, R. Lumry, Nature 1963, 200, 242-244; b) M. S. Healy, J. E. Hanson, J. Appl. Polym. Sci. 2007, 104, 360-364; c) T. Sanoa, A. Uchiyamab, T. Sagoc, H. Itagakia, Eur. Polym. J. 2017, 90, 114-121.

[23] Z. R. Grabowski, K. Rotkiewicz, Chem. Rev. 2003, 103, 3899-4031.

[24] V. S. Padalkar, S. Seki, Chem. Soc. Rev. 2016, 45, 169-2 
